\title{
Hybrid molecular electronic (HME) transistor based on deoxyguanosine derivatives
}

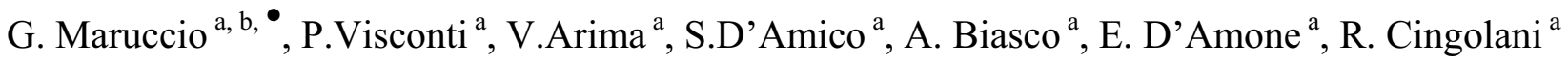 \\ and R. Rinaldi ${ }^{\mathrm{a}}$, S. Masiero ${ }^{\mathrm{c}}$, T.Giorgi ${ }^{\mathrm{c}}$ and G. Gottarelli ${ }^{\mathrm{c}}$ \\ ${ }^{a}$ National Nanotechnology Laboratory of INFM, Università of Lecce, 73100 Lecce, Italy \\ ${ }^{\mathrm{b}}$ Department of Physics, University of Lecce \\ ${ }^{c}$ Department of Organic Chemistry, University of Bologna, 40126 Bologna, Italy
}

\begin{abstract}
In this work, a field effect transistor based on deoxyguanosine derivatives (a DNA basis) is demonstrated by means of systematic transport experiments. Our nanodevices were fabricated starting from a deoxyguanosine derivative $\left(\mathrm{dG}\left(\mathrm{C}_{10}\right)_{2}\right)$ layer interconnecting planar nano-electrodes, with separation in the 20-40nm range. The three terminal devices exhibit a maximum voltage gain of 0.76 . Though the quick aging and the reproducibility of the devices have to be improved, the realization of a transistor-like device represents a starting point towards the development of planar solid-state bio-molecular electronic devices.
\end{abstract}

Keywords: Molecular electronics, three terminal devices, DNA bases

\section{INTRODUCTION}

Electronics is usually identified with metal-oxide-semiconductor circuits. From its beginning in the early 1970's, the semiconductor industry has accomplished a remarkable miniaturization trend, driven by the continuous downsizing of the components, such as MOSFET, thanks to the significant advances of the lithographic techniques (top-down approach). Better and cheaper has been the criterion of the success. But gaining the nanometer scale requires a turning point and conceptually new devices to overcome the physical and technological limitations of the current top-down approach in semiconductor electronic devices fabrication. Hybrid molecular devices, consisting of molecules between two electrodes, represent an interesting alternative in which molecular materials are chosen to perform the basic functions of digital electronics. The idea of developing a molecular electronics comes from a farsighted paper of Aviram and Ratner ${ }^{1}$. Recent works ${ }^{2,3,4,5,6,7}$ have shown that the concept of hybrid molecular devices is useful and practicable for individual components exploiting the advances in the synthesis of electronic molecules ${ }^{8}$ and the fabrication of metallic electrodes with nanometer-scale separation ${ }^{9},{ }^{10}$. However, a great challenge remains to move from prototypes to commercial devices, because of the intrinsic difficulty to interconnect molecules and to develop fully-reproducible and high-throughput fabrication process. Moreover, device performances have to be further improved to reach and surpass those of conventional electronic devices.

Here we report the realization of a field effect transistor based on a modified DNA base, where the molecular layer acts as semiconductor channels whose conductivity can be controlled by means of a gate electrode. The transistor characteristics present a threshold voltage, whose value can be tuned using the gate voltage to alter the alignment condition between the Fermi-level in the electrodes and the molecular miniband. We find that the maximum voltage gain and the on-off ratio are 0.76 and 5 respectively.

\footnotetext{
• giuseppe.maruccio@unile.it; phone+39 0832321 877; fax +39 0832326351
} 


\section{FABRICATION AND CHARACTERIZATION}

Recently, there has been a lot of theoretical and experimental work aimed to assess the problem of the electrical transport in DNA strands. Due to the considerable difficulties in connecting single DNA ropes to nanoelectrodes, we adopt a different strategy choosing a single modified DNA base, the lipophilic deoxyguanosine, as the basic molecular constituent of our nanodevices. The guanine exhibits a low oxidation potential -which favors charge transport- and spontaneous self-assembling properties both in solution and in the solid state ${ }^{11}$. The base was modified, in the lipophilic deoxyguanosine derivative form ${ }^{12}$ (Fig.1a), to favor the formation of ordered supramolecolar structures thanks to its peculiar sequence of H-bond donor and acceptor groups and side chains.

The nanodevices were fabricated by cast deposition. A $2 \mu \mathrm{l}$ drop of deoxyguanosine solution in chloroform was prepared $\left(10^{-1}-10^{-4} \mathrm{M}\right)$ and deposited in the gap between two metallic contacts. Upon controlled evaporation, Atomic Force Microscopy (AFM) studies of the guanosine layer showed that the deoxyguanosine molecules self-assembly in supramolecular ribbon-like structures (Fig. 1c).

(a)
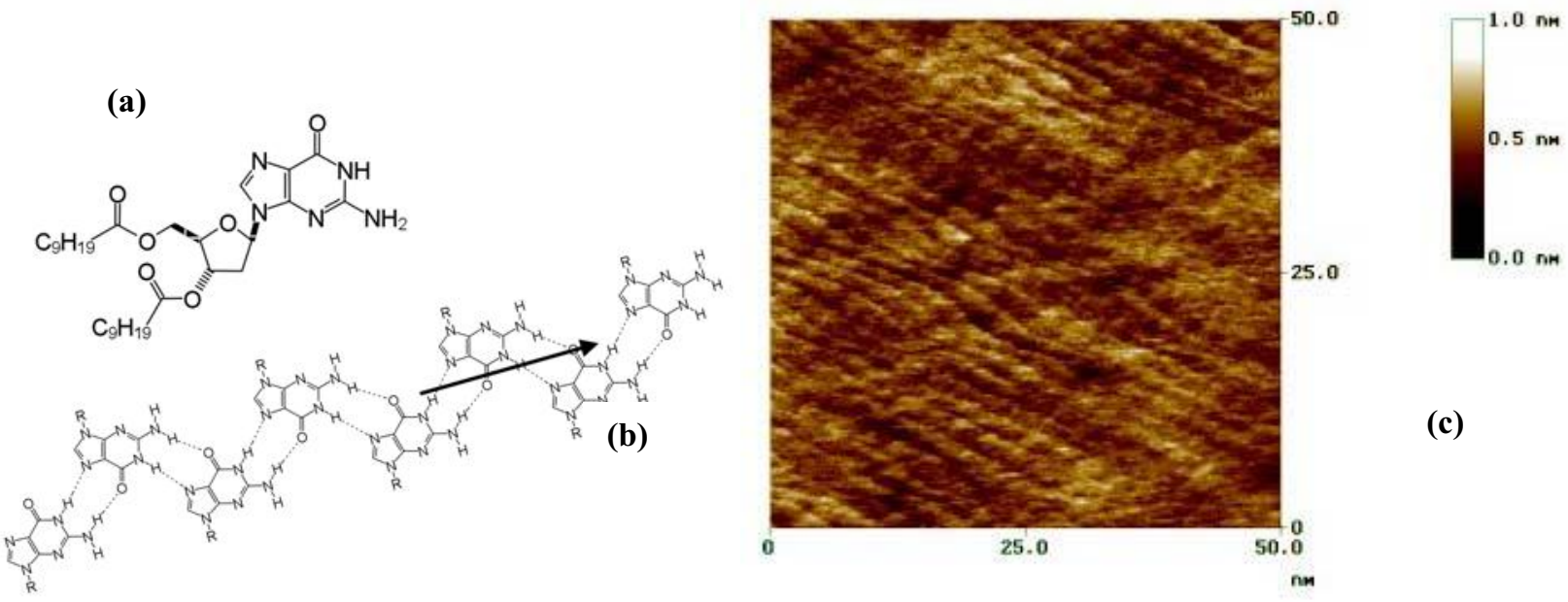

(c)

Figure $1:$ (a) The molecule used in this study and (b) the self organized ribbons of $\mathrm{dG}\left(\mathrm{C}_{10}\right)_{2}$. (c) AFM image of the guanosine layer morphology. The ribbons form an ordered supramolecular structure, laying parallel to each other with a periodicity of 2.5 $\mathrm{nm}$ and a length up to $l \approx 100 \mathrm{~nm}$. Over such a length scale, the packed lamellar structure of the SAGC, physisorbed on the surface between the electrodes, gives rise to an orthorombic unit cell of size $a=1.3 \pm 0.1 \mathrm{~nm}$ and $b=2.5 \pm 0.2 \mathrm{~nm}$. For distances longer than $100 \mathrm{~nm}$, that ordering is lost, and the ribbons form randomly oriented SAGCs.

To interconnect guanosine-ribbons and implement the hybrid molecular electronic devices, planar metal-insulator-metal nanojunctions (Fig. 2) were fabricated on the oxide surface of thermally oxidized silicon wafers. The oxide layer was $100 \mathrm{~nm}$ thick and constituted the gate oxide, while a silver back-electrode-deposited on the degenerated doped silicon substrate- was chosen as gate electrode. The source and drain $\mathrm{Cr} / \mathrm{Au}(8 \mathrm{~nm} / 15 \mathrm{~nm})$ electrodes were realized by electron beam lithography followed by lift-off. Typical inter-electrodes distances were in the range between 20 and $40 \mathrm{~nm}$. The fabricated nanodevices were inspected by plan-view and cross-section scanning electron microscopy (SEM) in order to check the success of the whole technological process and to estimate the separation between the $\mathrm{Cr} / \mathrm{Au}$ nanotips. All nanojunctions were then tested by current-voltage measurements. Open-circuit resistance as high as 100-200G $\Omega$ were obtained when the technological process succeeded. Typical process yield - i.e. the percentage of good nanojunctions where no significant current was observed to flow under open-circuit configuration - is around $90 \%$.

Current-voltage experiments were focused on the DC behaviour and were carried out by using a semiconductor parameter analyser (HP Agilent 4155B) in the voltage range between -3.5 and $3.5 \mathrm{~V}$ at room temperature and in air. The gate and drain voltage are applied with respect to the grounded electrode (source). Drain-source and gate current are recorded simultaneously. Fig. 3 shows a 3D plot of the drain-source current as a function of the drain-source voltage $\mathrm{V}_{\mathrm{ds}}$ and the gate voltage $\mathrm{V}_{\mathrm{g}}$. In the low drain-source bias region, the device was almost no conductive. All the measured 
characteristics showed the existence of a well-defined threshold voltage and indicated that conduction can be activated at higher bias. By increasing the gate voltage from $0 \mathrm{~V}$ to $1 \mathrm{~V}$, this threshold voltage of the characteristic gradually shifted to higher values following an almost linear trend. This behaviour suggests that the transport mechanism is due to resonant tunneling.

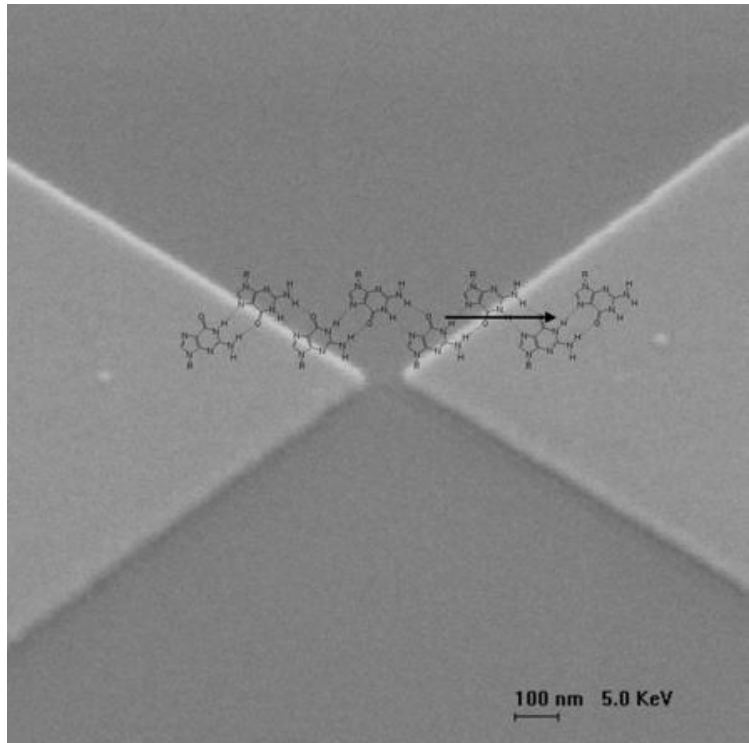

Figure 2: High magnification SEM image of the planar nanoelectrodes.

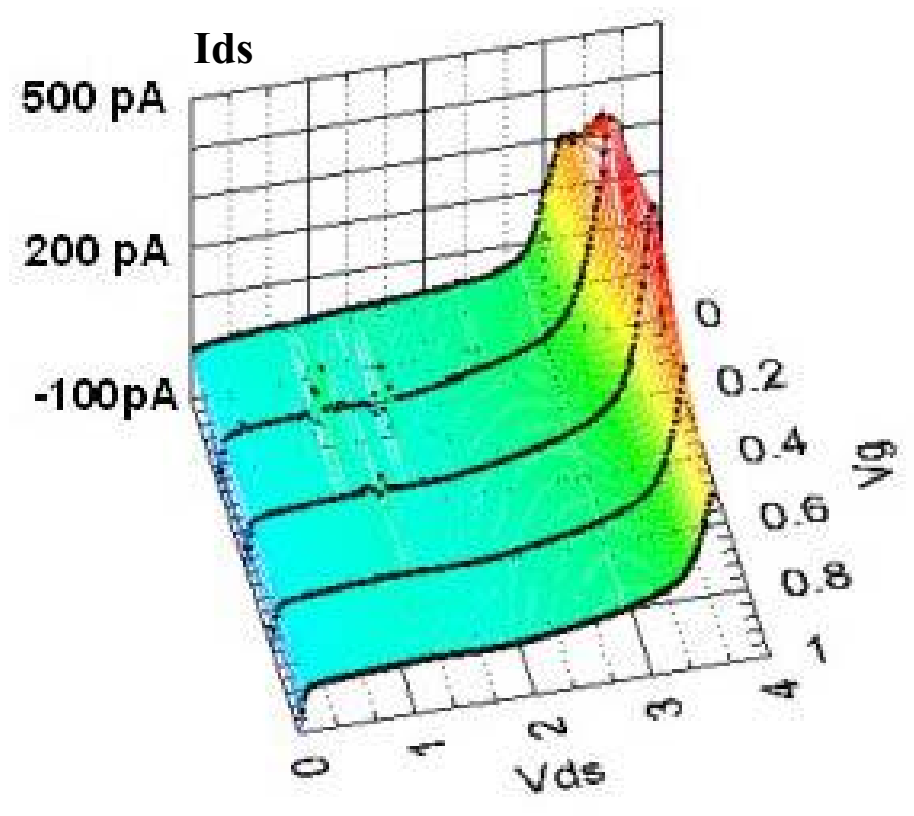

Figure 3: 3D plot of the drain-source current as a function of the drain-source voltage $\mathrm{V}_{\mathrm{ds}}$ and the gate voltage $\mathrm{V}_{\mathrm{g}}$

Due to the difficulty in creating an ideal Ohmic molecole-metal contact ${ }^{13,6}$, two potential barriers arise at the interfaces between the organic/inorganic layers. Moreover, since the molecules were found to aggregate spontaneously to form ordered self-assembled guanosine crystals (SAGCs), we could model the molecular part as a semiconductor material, characterized by energy bands. At low voltage, the molecular layer behaved as an insulator. On the other hand, resonant tunneling was the dominating transport mechanism at and above threshold. When the applied drain-source bias aligned the electrodes Fermi levels to one molecular layer miniband, conduction was activated. The shift in the energy of the molecular band due to the field-effect induced a change in the alignment condition and therefore an increase in the threshold voltage, was required to recover the resonant tunneling process. We found that the threshold voltage increased almost linearly with the gate voltage.

The field effect was found to have a profound effect on the drain-source current at constant drain-source voltage, as manifested by a fivefold decrease of its value as a function of $\mathrm{V}_{\mathrm{g}}$ (for drain-source voltage around $3 \mathrm{~V}$, the current $\mathrm{I}_{\mathrm{ds}}$ changes from $250 \mathrm{pA}$ at $\mathrm{V}_{\mathrm{g}}=0 \mathrm{~V}$ to $50 \mathrm{pA}$ at $\mathrm{V}_{\mathrm{g}}=1 \mathrm{~V}$ resulting in an on-off ratio around 5). It is worth noting that $\mathrm{I}_{\mathrm{ds}}$ decreases as in a p-channel MOSFET, but no saturation was observed in the $\mathrm{I}_{\mathrm{ds}}-\mathrm{V}_{\mathrm{ds}}$ characteristics. A maximum voltage gain as high as 0.76 was found (small-channel molecular devices ${ }^{6,7}$ usually exhibit gain in the range between 0.3 and 0.5). The gate current is as low as few $\mathrm{pA}$ and a negligible dependence on $\mathrm{V}_{\mathrm{ds}}$ was observed (fig.4). As expected, in fact, the gate current only depends on the gate voltage and we observe almost parallel characteristics. From the slopes of the $\mathrm{I}-\mathrm{V}_{\mathrm{ds}}$ curves, we can see that the derivative conductance of the deoxyguanosine transistor after threshold increases with $V_{d s}$. Fig. 5 displays resistance $R_{d s}-V_{d s}$ characteristics at different gate voltage. Five $R_{d s}-V_{d s}$ curves at $V_{g}=0.00,0.25$, $0.50,0.75,1.00 \mathrm{~V}$ are displayed in this figure. At first, the resistance is as high as tens of $\mathrm{G} \Omega$ and then gradually decreases to reach few $\mathrm{G} \Omega$ near threshold. Its value increases with the gate voltage. 


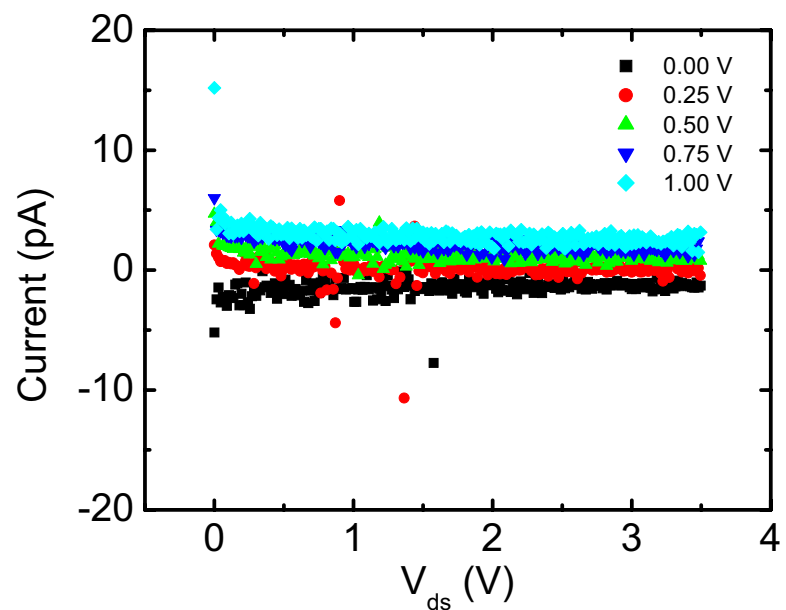

Figure 4: The gate current is as low as few pA and shows a negligible dependence on the drain-source voltage.

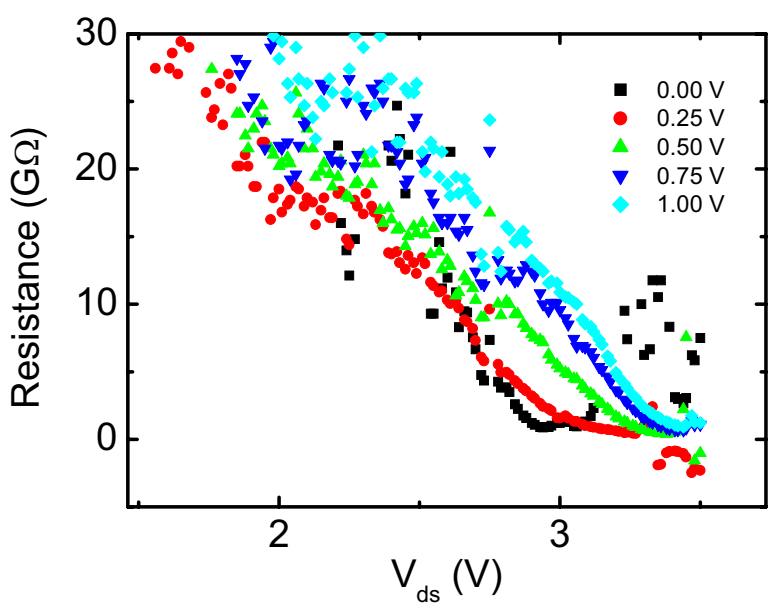

Figure 5: Dependence of the drain-source resistance on the voltage at discrete gate voltages.

Although the nanodevices could be probably further improved, by enhancing their performance and reducing their ageing, some interesting architecture can already be proposed. The lack of saturation in the source-drain current makes the output current quite sensitive to small changes in the source-drain voltage and gives rise to the issues of stability against drift. If the operation conditions are changed a decrease of both output resistance and gain can be generated. In any case, the behaviour of our nanodevices is dual with respect to a standard field-effect transistor. In fact, the $V_{\mathrm{ds}}-I_{\mathrm{ds}}$ is similar to the $\mathrm{I}_{\mathrm{ds}}-\mathrm{V}_{\mathrm{ds}}$ curve of a standard FET (fig.6). Our devices is stable when guided in current rather than in voltage. Moreover, an on-off ratio around 5 could be enough for some logic applications, such as static random access memory (S-RAM). This is a good result since other molecular-device prototypes exhibit similar values ${ }^{5}$. Improving performances requires to avoid leakages and to reduce the injection barriers at the metal-molecules interface in order to create an ideal Ohmic contact.

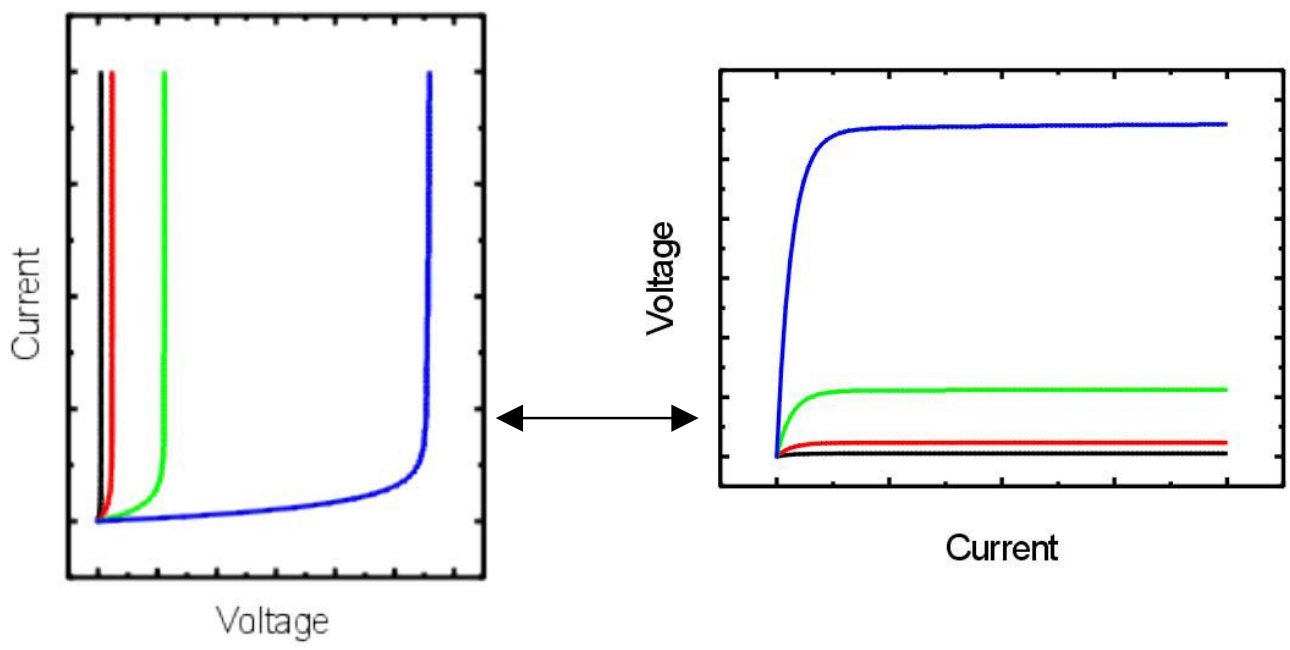

Figure 6: Duality between the behaviour of our nanodevices and a standard field-effect transistor. The V-I characteristics of our nanodevices are similar to the I-V characteristics of a standard field-effect transistor. 


\section{CONCLUSIONS}

In conclusion, a HME transistor based on a deoxyguanosine derivative and operating at room temperature and ambient condition has been demonstrated. Conduction was explained in terms of band alignment and resonant transport in the molecular layer. Although the economic fabrication of complete circuits at the molecular level remains challenging due to the difficulty to interconnect molecules and the ageing issue, the realization of the concept of a three terminal devices exhibiting modulation is a key result for molecular electronics. Our nanodevices are interesting since they operate on the nanometer scale and can be used to pursuit the miniaturization trends. Moreover, the possibility to control the threshold condition by means of the gate voltage allow different applications.

\section{REFERENCES}

[1] A.Aviram, M.A. Ratner, "Molecular rectifiers", Chem. Phys. Lett., 29, 277-283, 1974

[2] C. Joachim, J.K.Gimzewski and A.Aviram, "Electronics using hybrid-molecular and mono-molecular devices", Nature, 408, 541-548, 2000

[3] G. Maruccio, P.Visconti, V.Arima, S.D'Amico, A.Biasco, E.D’Amone, R.Cingolani, R.Rinaldi, S.Masiero, T.Giorgi, G.Gottarelli, "Field Effect Transistor Based on a Modified DNA Base", NanoLetters, 3, 479-483, 2003

[4] Jorge M. Seminario, A.G.Zacarias and J.M.Tour, "Theoretical Study of a Molecular Resonant Tunneling Diode", J.Am.Chem.Soc., 122, 3015-3020, 2000

[5] M.A. Reed, J.Chen, A.M.Rawlett, D.W.Price and J.M.Tour, "Molecular random access memory cell", Appl.Phys.Lett., 78, 3735-3737, 2001

[6] J. Appenzeller, J. Knoch, V. Derycke, R. Martel, S. Wind and Ph. Avouris, "Field-Modulated Carrier Transport in Carbon Nanotube Transistors", Phys.Rev.Lett., 89, 126801, 2002

[7] S. J. Tans, Alwin R. M. Verschueren and Cees Dekker, "Room-temperature transistor based on a single carbon nanotube", Nature, 393, 49-52, 1998

[8] J.M. Tour, "Molecular electronics. Synthesis and testing of components", Acc. Chem. Res., 33, 791-804, 2000

[9] S. Cholet, C.Joachim, J.P. Martinez, B.Rousset, "Fabrication of co-planar metal-insulator-metal solid state nanojunctions down to 5 nm", Eur. Phys. J. AP, 8, 139-145, 1999

[10] Y.V Kervennic, H.S.J. Van der Zant, A.F.Morpurgo, L. Gurevich, L.P. Kouwenhoven, "Nanometer-spaced electrodes with calibrated separation", Appl. Phys. Lett., 80, 321-323, 2002

[11] G.Gottarelli, S.Masiero, E.Mezzina, G.P.Spada, P.Mariani and M.Recanatini, Helv.Chim.Acta, 81, 2078, 1998

[12] G.Gottarelli, S.Masiero, E.Mezzina, S.Pieraccini, J.P.Rabe, P.Samorì and G.P.Spada, Chem.Eur.J., 6, 3242, 2000

[13] PRL Malenfant, CD Dimitrakopoulos, JD Gelorme, LL Kosbar, TO Graham, A Curioni, W Andreoni, “ $N$-type organic thin-film transistor with high field-effect mobility based on a $\mathrm{N}, \mathrm{N}^{*}$-dialkyl-3,4,9,10-perylene tetracarboxylic diimide derivative", Appl.Phys.Lett., 80, 2517-2519, 2002 